\title{
EFEITO DO SUBSTRATO E LUMINOSIDADE NA GERMINAÇÃO DE Anadenanthera colubrina (FABACEAE, MIMOSOIDEAE) ${ }^{1}$
}

\author{
Ana Carolina da Cunha Rodrigues ${ }^{2}$, Juan Tomás Ayala Osuna ${ }^{3}$, Sandra Regina de O. D. Queiroz e Ana
} Paula Souza Rios ${ }^{5}$

\begin{abstract}
RESUMO - Angico (Anadenanthera colubrina (Vell.) Brenan var. cebil (Griseb.) Altschul) é uma Mimosaceae arbórea de grande distribuição na América do Sul, com rápido crescimento e bastante utilizada para fornecimento de tanino, madeira, mel e resina, além de ser considerado ornamental e medicinal. O objetivo deste trabalho foi analisar comparativamente a germinação de sementes procedentes de populações de uma mesma espécie de dois locais com climas distintos. Foram observados a germinabilidade $(\% \mathrm{G})$, o tempo médio (t) e o índice de velocidade de germinação (IVG) em germinador a $30^{\circ} \mathrm{C} \pm 1$ e emergência em viveiros com diferentes tipos de substratos e luminosidades. O ambiente a pleno sol proporcionou as menores taxas de germinação, maiores tempos médios e menores índices de velocidade de germinação em todos os tipos de solos. As luminosidades mais adequadas são as de $30 \%$ de luz no substrato areia e $50 \%$ de luz na terra vegetal pura. A população da cidade de Tanquinho-BA, pelo seu vigor, mostrou-se mais adaptada para estabelecimento em campo, aproveitando condições favoráveis ao subseqüente desenvolvimento. Esses comportamentos germinativos diferenciados evidenciam diferenças adaptativas aos "habitats" onde ocorrem contribuindo para o sucesso ecológico e evolutivo da espécie.
\end{abstract}

Palavras-chave: Germinabilidade, substrato, luminosidade, vigor e ecofisiologia.

\section{EFFECT OF SUBSTRATE AND LUMINOSITY ON THE GERMINATION OF Anadenanthera colubrina (FABACEAE, MIMOSOIDEAE)}

\begin{abstract}
Angico (Anadenanthera colubrina (Vell.) Brenan var. cebil (Griseb.) Altschul) is a fast growing Mimosaceae, widely distributed in South America, used for production of tannin, wood, honey, resin, besides being ornamental and medicinal. The objective of this work was to analyze the germination of seeds coming from populations of a same species from two sites with different climates. Germinability $(\% G)$, average time $(t)$ and germination velocity index $(G V I)$ were studied in germination chambers at $30 \pm 1{ }^{\circ} \mathrm{C}$ and nurseries with different types of substrates and luminosities. Total luminosity provided the lowest germination index, longest average time and lowest germination velocity index in all tested substrates. The most suitable luminosities are $30 \%$ of light for the sand substrate and $50 \%$ of light for the natural substrate. The population from the municipality of Tanquinho - BA was shown more adapted for establishment in field, for its vigor, taking advantage of favorable conditions to the subsequent development. These distinct germinative behaviors suggest adaptation to the different habitats in which they occur, contributing to the species ecological and evolutionary success.
\end{abstract}

Keywords: Germinability, substrate, luminosity, vigorous and ecophysiology.

\footnotetext{
${ }^{1}$ Recebido em 18.12.2005 e aceito para publicação em 26.03.2006.

${ }^{2}$ Bolsista DTI/CNPq, Mestre em Botânica pela Universidade Estadual de Feira de Santana (UEFS), endereço para correspondência: R. Alfredo Passos, 430, Bairro Ana Lúcia, 44380-000 Cruz das Almas, Bahia. E-mail:<acreco@ig.com.br >.

${ }^{3}$ Departamento de Ciências Biológicas da UEFS. Horto Florestal, Av. Santa Mônica, S/N, 44100-000 Feira de Santana, Bahia. ${ }^{4}$ PRODOC/FAPESB/CNPq.

${ }^{5}$ Bolsista FAPESB.
} 


\section{INTRODUÇÃO}

Anadenanthera colubrina var. cebil (Mimosaceae), vulgarmente conhecida por angico, está entre as espécies nativas do Semi-Árido que se encontram em risco de extinção, apesar de ser uma espécie de ampla ocorrência, facilmente adaptada a diversos tipos de ambiente em vários países da América do Sul. Possui grande demanda no mercado, sendo utilizada como planta ornamental, fornecedora de tanino e mel, como forrageira, energética, resinífera, madeireira e, inclusive, medicinal. Esse uso intenso, até por indústrias, coloca em risco a existência dessa e de outras espécies, aliado à ausência de cultivos ou de métodos de propagação, ao desconhecimento da real distribuição das populações nativas e à ausência de estudos que avaliem o impacto do extrativismo na estrutura e biologia dessas populações (ALBUQUERQUE e ANDRADE, 2002).

É de grande importância o estudo da germinação de sementes de espécies do Nordeste brasileiro para definir práticas de manejo, desenvolver novas técnicas para produção de mudas e possibilitar melhor aproveitamento, o que se torna o ponto de partida para utilização e exploração de forma racional das espécies nativas, especialmente da Caatinga, cujos trabalhos sobre germinação ainda são escassos Cabral et al. (2003). A utilização de espécies nativas na reabilitação ambiental não tem sido empregada em virtude da ausência de conhecimento consolidado sobre a biologia, ecologia, técnicas de propagação e manejo dessas espécies (RANIERI et al., 2003). As sementes constituem a via de propagação mais empregada na implantação de plantios, e a busca de conhecimentos sobre as condições ótimas para os testes de germinação desempenha papel fundamental dentro da pesquisa, além de fornecer informações valiosas sobre a propagação de espécies (VARELA et al., 2005).

A germinação é o fenômeno pelo qual, em condições adequadas de temperatura, água e oxigênio, o eixo embrionário continua seu desenvolvimento, que havia sido interrompido, nas sementes ortodoxas, pela maturidade fisiológica (CARVALHO e NAKAGAWA, 2000). E em situações naturais, as sementes estão submetidas a uma série de pressões, como variações na umidade do solo, radiação, competição e, inclusive, ataque de fungos patogênicos, condições desfavoráveis para expressar todo seu potencial germinativo (CARVALHO e NAKAGAWA, 2000; HILHORST et al.,

\section{R. Árvore, Viçosa-MG, v.31, n.2, p.187-193, 2007}

2001; DHINGRA et al., 2002). Conforme Brasil (1992), a germinação de sementes é a emergência e o desenvolvimento das estruturas essenciais do embrião, que mostram estar apto para produzir uma planta normal sob condições favoráveis de campo.

Se sementes de uma mesma espécie apresentam germinabilidade semelhante, não quer dizer que ambas exibem o mesmo comportamento, pois o início da germinação e a sua distribuição podem ser diferentes, apresentando maior ou menor rapidez (FERREIRA et al., 2001; BORGHETTI e FERREIRA, 2004). Os testes mais simples para determinação de vigor são os de velocidade de desenvolvimento, sendo os mais utilizados o tempo médio de germinação e o índice de velocidade de germinação, que se baseiam no pressuposto de que sementes mais vigorosas germinarão mais rapidamente do que outras em condições inferiores, distinguindo as sementes de um mesmo lote (PIÑA-RODRIGUES et al., 2004).

Durante a produção de mudas, o substrato é um dos componentes que mais interferem na sua germinação e no crescimento, por meio de fatores como estrutura e textura. Às vezes, as características físicas do solo tornam-se tão importantes quanto as propriedades químicas, pois a melhor aeração e permeabilidade de substratos mais arenosos promovem a menor incidência de microrganismos que podem interferir negativamente no processo germinativo, além de fornecer oxigênio e água para as sementes, acelerando a taxa de germinação e reduzindo a necessidade de desinfestação, como recomendou Nobre (1994).

O conhecimento da biologia de sementes de espécies filogeneticamente relacionadas, mas que ocupam microhábitats distintos, é importante para a compreensão de processos comunitários como sucessão, estabelecimento de plântulas e regeneração natural (RANIERI et al., 2003). Botezelli et al. (2000) destacaram a importância de se trabalhar com plantas de diferentes localidades, constatando diferentes expressões fenotípicas devido a variações de temperatura, fotoperíodo e precipitação, entre outros fatores que ressaltam determinados aspectos da composição genética e que não se mostrariam em outros ambientes, captando, assim, expressões do genótipo, possibilitadas pelas condições ambientais adequadas. Sabe-se que essa espécie de angico se adapta muito bem a diversos ambientes, e, em razão disso, algumas características podem variar 
ou desaparecer e ser substituídas. Laboriau (1983) citou que a extensa distribuição geográfica de uma espécie é devida à ampla faixa de temperatura para germinação, importante para a ecologia de populações e denota caráter adaptativo que propicia capacidade de estabelecimento em campo.

Foram encontrados diversos trabalhos sobre germinação de A. colubrina (BARBOSA, 1980; BARBOSA, 1992; OLIVEIRA, 1997; SILVA, 1998; SILVA e BARBOSA, 2000; BARBOSA, 2003; SILVAet al., 2004), mas nenhum deles fez uma análise comparativa da germinação de sementes procedentes de populações dessa mesma espécie de dois locais de climas distintos, o que levou ao objetivo deste trabalho. Tal análise se torna importante para caracterizar fisiologicamente as diferentes populações pelo tempo, pela porcentagem e pelo índice de velocidade da germinação em condições de laboratório e de viveiro utilizando diferentes tipos de substrato e luminosidades, que refletem adaptações ecológicas aos dois ambientes onde as populações vivem.

\section{MATERIAL E MÉTODOS}

Os frutos maduros de $A$. colubrina var. cebil foram coletados na Fazenda Floresta, localizada na cidade de Tanquinho, BA e em terreno da Escola de Agronomia da Universidade Federal da Bahia (UFBA), em Cruz das Almas, BA, em outubro/novembro de 2003 e setembro/outubro de 2004, de indivíduos adultos de cada população.

A cidade de Tanquinho, BA, localizada a 11'52'30'S e 3907'30'W, possui clima típico de Semi-Árido Baiano com vegetação de contato caatinga-floresta estacional e floresta estacional decidual, temperaturas médias variando entre 18 e $30^{\circ} \mathrm{C}$ e precipitação média anual de 500 a 800 mm (ANUÁRIO..., 2004). Já a cidade de Cruz das Almas, BA, localizada a $12^{\circ} 41^{\prime} 15^{\prime}$ 'S e $39^{\circ} 03$ ' $45^{\prime \prime} \mathrm{W}$, exibe clima tropical úmido a subúmido, com vegetação de floresta estacional decidual e semidecidual, temperaturas médias alternando entre 19 e $28^{\circ} \mathrm{C}$ com médias de chuvas anuais de $1.200 \mathrm{~mm}$ (ANUÁRIO..., 2004).

Os experimentos foram iniciados logo após a retirada das sementes de dentro dos frutos, descartando-se as extremamente pequenas, enrugadas ou predadas.

Em laboratório, os ensaios foram realizados em câmara de germinação a $30 \pm 1^{\circ} \mathrm{C}$, com fotoperíodo de 12 horas, em caixas plásticas transparentes do tipo
Gerbox com tampa e dupla camada de papel germitest, umedecidos com água destilada e devidamente esterilizados. Cada Gerbox continha 10 sementes, aparentemente intactas e viáveis, todas tratadas previamente com solução de hipoclorito de sódio $2 \%$ por 5 min e lavadas em água destilada, em delineamento inteiramente casualizado (DIC) com 10 repetições.

Nos viveiros, cobertos com sombrite, as sementes foram plantadas diretamente em sacos de polietileno contendo uma única semente no primeiro centímetro das distintas misturas de solos, de acordo com Ramos et al. (1982). Essas misturas de solos apresentavam ordem crescente do grau de porosidade: solo 1 - terra vegetal, solo 2 - terra vegetal+areia (3:1), solo 3 - terra vegetal+areia (1:1), solo 4 - areia+terra vegetal (3:1) e solo 5 - areia pura. Foram submetidos a diferentes intensidades luminosas (L1 - 100\% de luz (a pleno sol), L2 - 50\% e L3 - 30\%), constituindo um arranjo fatorial $2 \times 5 \times 3$ e tratamentos estruturados, no delineamento inteiramente casualizado com 10 a 15 repetições por tratamento.

As parcelas foram regadas duas vezes ao dia, de acordo com a capacidade de campo dos solos, e a capina foi manual, quando necessária. Os experimentos duraram no máximo 13 dias e foram finalizados quando não havia mais germinação e as sementes apresentavam sinais de deterioração. Foi considerada germinada a semente que se apresentava conforme o conceito de ANUÁRIO..., (1992), apresentando-se como plântula normal.

A geminabilidade $(\% \mathrm{G})$ representa o número total de sementes germinadas sob determinada condição experimental e foi calculada de acordo com Borghetti e Ferreira (2004), pela fórmula $\% \mathrm{G}=\left(\Sigma \mathrm{n}_{\mathrm{i}} \cdot \mathrm{N}^{-1}\right) \cdot 100$, em que $\Sigma n_{\mathrm{i}}$ corresponde ao número total de sementes germinadas em relação ao número de sementes dispostas para germinar $(\mathrm{N})$.

Para quantificar a germinação sob o ponto de vista cinético, o tempo médio de germinação $(\mathrm{t})$ foi calculado pela equação $t=\Sigma n_{i} \cdot t_{i} / \Sigma n_{i}$, em que $n_{i}$ é o número de sementes germinadas dentro de determinado intervalo de tempo $t_{\mathrm{i}-1}$ e $\mathrm{t}_{\mathrm{i}}$ (BORGHETTI e FERREIRA, 2004).

Oíndice de velocidade de germinação (IVG) evidencia o número de sementes germinadas a cada dia e expressa diretamente o vigor delas pela fórmula de Maguire (1962), em que $I V G=G_{1} / N_{1}+G_{2} / N_{2}+\ldots G_{n} / N_{n}$, na qual $G_{1}, G_{2}$ $\ldots \mathrm{G}_{\mathrm{n}}$ é igual ao número de sementes germinadas, $\mathrm{e}$ $\mathrm{N}_{1}, \mathrm{~N}_{2} \ldots \mathrm{N}_{\mathrm{N}}$ corresponde ao número de dias.

R. Árvore, Viçosa-MG, v.31, n.2, p.187-193, 2007 
As porcentagens de germinação de sementes de cada repetição foram previamente transformadas em arcoseno $\sqrt{P / 100}$, para normalização dos dados e estabilização das variâncias de tratamentos (SANTANA e RANAL, 2004). A análise de variância foi feita pelo Sisvar e as médias, comparadas pelo teste de Tukey a $1 \%$ de probabilidade. Em casos de interação luminosidade e substrato significativa, houve a decomposição também analisada pelo Sisvar e médias comparadas pelo teste de Tukey a $1 \%$.

\section{RESULTADOS E DISCUSSÃO}

As porcentagens de germinação nos viveiros mostraram-se estatisticamente similares, fato que não ocorreu em laboratório, onde os melhores resultados foram apresentados pelas sementes trazidas da população de Tanquinho, BA, com diferença significativa a 1\%. Chegouse a uma média de $89 \%$ contra $38 \%$ das sementes trazidas de Cruz das Almas, com grande contaminação em laboratório desta última (Figura 1). Oliveira (1997) obteve $82 \%$ de germinação em germinador a $25^{\circ} \mathrm{C} \pm 1$ sob iluminação constante e $52 \%$ em substrato composto por terra e areia (1:1), mantido em sombrite a $50 \%$.

O fato de as sementes da população de Cruz das Almas apresentarem maior incidência de contaminação em laboratório pode ter contribuído para um reduzido porcentual de emergência, apesar de terem sido usados procedimentos usuais para esterilização. Segundo Dhingra et al. (2002), fungos patogênicos de Anadenanthera macrocarpa reduzem a emergência de plântulas e, conseqüentemente, a qualidade de crescimento de sementes contaminadas, e ao mesmo tempo a colonização inicia-se durante a formação das sementes, logo após a constituição do pericarpo, e continua a aumentar até a maturidade. Nobre (1994) não encontrou diferenças significativas entre médias de germinação de sementes tratadas com diversos métodos de desinfestação; apenas a utilização de Benomil permitiu maior expressão do vigor, mas o autor recomenda a não-desinfestação dos frutos e a simples secagem das sementes em estufa a $35^{\circ} \mathrm{C}$ por 18 horas, como tratamento mais promissor, devido ao menor número de plântulas anormais.

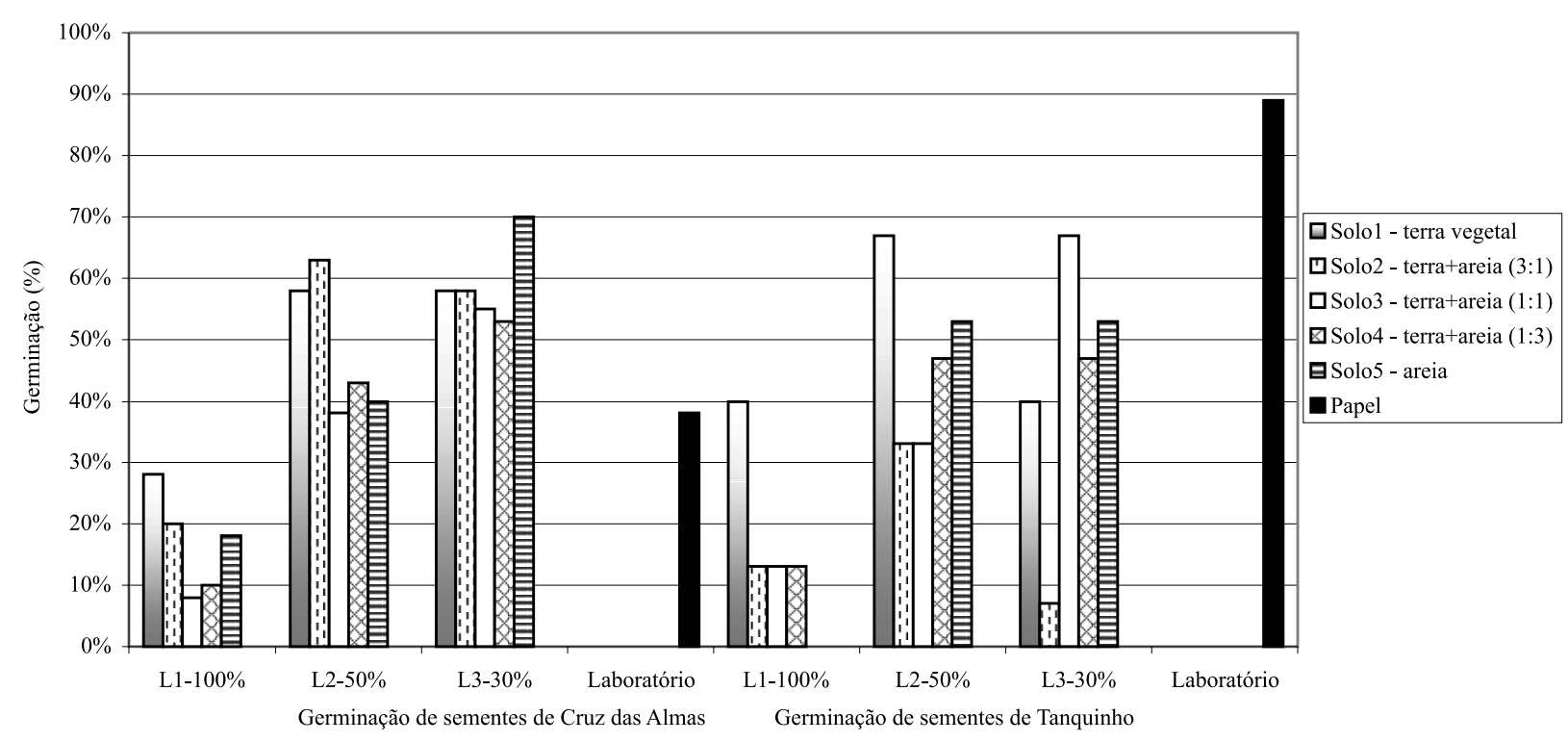

Figura 1 - Distribuição da porcentagem da germinação de sementes de A. colubrina var. cebil procedentes de Cruz das Almas e Tanquinho, BA, germinadas em laboratório e viveiros e submetidas a diferentes condições de substrato e luminosidade $-2003 / 2004$.

Figure 1 - Distribution of seed germinability of A. colubrina var. cebil derived from Cruz das Almas and Tanquinho - BA and germinated in different conditions of substrate and luminosity - 2003/2004.

R. Árvore, Viçosa-MG, v.31, n.2, p.187-193, 2007 
O maior grau de intensidade dessa associação biológica apresentada pelas sementes coletadas em Cruz das Almas, e muito mais reduzida pela população de Tanquinho, pode ser explicada pelo fato de aquelas populações estarem inseridas em ambientes com diferentes umidades relativas, temperaturas e pluviosidades, entre outros fatores, já terem vindo contaminadas com maior ou menor intensidade, em que uma simples imersão em hipoclorito de sódio não foi suficiente para sua desinfestação. Segundo Carvalho e Nakagawa (2000), existem raças fisiológicas patogênicas que exibem forte seleção preferencial em relação a um hospedeiro ou variedade, ou no caso de uma população inserida em um específico ecossistema. Isso é explicado pela resistência natural de plantas a certos patógenos, demonstrando nulidade ou menor intensidade de infestação. Além disso, são citados fatores químicos e físicos, como teor de água, temperatura, acidez, nutrientes, potencial de inóculo, organismos antagônicos presentes na microflora e tipo de germinação, que também podem auxiliar o estabelecimento do patógeno.

Os desdobramentos das interações nos diferentes solos (3, 4 e 5), contendo misturas 1:1 e 1:3 de areia + terra vegetal e apenas areia, respectivamente, demonstraram que a luz prejudica a germinação, e por isso há a necessidade de adensamento populacional dos indivíduos em campo. Há uma tendência de regressão linear comprovando que, quanto menor a incidência luminosa, melhor a germinação e maior é o poder germinativo. Possivelmente, o fator que interferiu foi a temperatura ambiente, modificada pela intensidade luminosa, juntamente com o tipo de solo. De acordo com Barbosa (1980; 2003), as sementes de A. colubrina var. cebil têm rápida germinação, são fotoblásticas neutras, com faixa ótima de temperatura entre 30 a 40 ${ }^{\circ} \mathrm{C}$, com 98-100\% de germinação. Em campo, na área de estudo Barbosa (1980) conseguiu 70-75\% de germinação após sete dias de observação.

A terra vegetal demonstrou ser o melhor substrato, devido a uma maior capacidade de retenção de água, com exceção da luminosidade 3 (30\% de luz) que mostrou melhor resultado no solo 5 (areia pura). Silva et al. (2004) encontraram melhores porcentagens de emergência, número médio de dias para a primeira emergência e índice de velocidade de emergência (IVE) em areia e profundidade superficial. Entretanto, no presente trabalho, para os testes de laboratório, a luz foi necessária ao desenvolvimento inicial da plântula, já que foi considerado o processo de germinação conceituado pela RAS (BRASIL, 1992).

A rapidez para o início de germinação, o tempo médio e IVG das sementes apresentaram diferença significativa a 1\%, em laboratório (Quadro 1). Com relação aos viveiros, houve diferença entre populações e luminosidades também a $1 \%$ de significância, sendo a incidência de luz total a que possuiu maior tempo médio, principalmente para as sementes de Cruz das Almas, que levaram de 6 a 10 dias para iniciar a germinação.

Quadro 1 - Características germinativas de sementes de A. colubrina var. cebil coletadas em Cruz das Almas e Tanquinho, BA - 2003/2004

Table 1 - Germinative characteristics of A. colubrina var. cebil seeds collected in Cruz das Almas and Tanquinho - BA-2003/2004

\begin{tabular}{|c|c|c|c|c|c|c|}
\hline \multirow[t]{2}{*}{ Tratamentos } & \multicolumn{2}{|c|}{$\begin{array}{l}\text { Tempo para início de } \\
\text { germinação (dias) }\end{array}$} & \multicolumn{2}{|c|}{$\begin{array}{l}\text { Tempo médio de } \\
\text { germinação (dias) }\end{array}$} & \multicolumn{2}{|c|}{$\begin{array}{c}\text { IVG } \\
\left(\mathrm{n}^{\mathrm{o}} \text { sementes/dia }\right)\end{array}$} \\
\hline & Cruz das Almas & Tanquinho & Cruz das Almas & Tanquinho & Cruz das Almas & Tanquinho \\
\hline Câmara de germinação & 5 & 3,5 & $4,45 * *$ & $6,41 * *$ & $0,23 * *$ & $0,16 * *$ \\
\hline Luz 1 solo 1 & 8 & 4 & 9,27 & 5,00 & 0,11 & 0,20 \\
\hline Luz 1 solo 2 & 8 & 5 & 8,88 & 5,50 & 0,11 & 0,18 \\
\hline Luz 1 solo 3 & 10 & 5 & 10,7 & 5,00 & 0,09 & 0,20 \\
\hline Luz 1 solo 4 & 7 & 5 & 8,50 & 5,00 & 0,12 & 0,20 \\
\hline Luz 1 solo 5 & 6 & - & 9,14 & - & 0,11 & - \\
\hline Luz 2 solo 1 & 5 & 3 & 9,17 & 4,60 & 0,11 & 0,22 \\
\hline Luz 2 solo 2 & 3 & 3 & 5,40 & 3,60 & 0,19 & 0,28 \\
\hline Luz 2 solo 3 & 4 & 3 & 5,47 & 3,60 & 0,18 & 0,28 \\
\hline Luz 2 solo 4 & 3 & 3 & 5,41 & 4,33 & 0,19 & 0,23 \\
\hline Luz 2 solo 5 & 3 & 3 & 5,44 & 3,88 & 0,18 & 0,26 \\
\hline Luz 3 solo 1 & 4 & 3 & 5,96 & 3,83 & 0,17 & 0,26 \\
\hline Luz 3 solo 2 & 3 & 4 & 5,22 & 4,00 & 0,19 & 0,25 \\
\hline Luz 3 solo 3 & 3 & 3 & 4,73 & 4,20 & 0,21 & 0,24 \\
\hline Luz 3 solo 4 & 4 & 3 & 4,71 & 3,43 & 0,21 & 0,29 \\
\hline Luz 3 solo 5 & 3 & 3 & 5,04 & 3,67 & 0,20 & 0,27 \\
\hline
\end{tabular}


Ao realizar-se o desdobramento do fator luminosidade dentro dos tipos de solos, a incidência total de luz continuou sendo diferente significativamente dentro de todos os substratos, também com tendência de regressão linear, com a menor luminosidade tendo o menor tempo médio de germinação. Isso pode ser explicado devido ao fato de que a plântula é bastante sensível à incidência direta de luz, associada à elevada temperatura, e no ambiente natural ela cresce protegida pela sombra da planta-mãe.

Quanto menor o tempo médio, mais vigorosa a amostra, e o menor tempo médio foi obtido pelas sementes procedentes de Tanquinho. Pode-se inferir que essa rapidez na germinação é uma estratégia para se estabelecer no ambiente o mais rápido possível, aproveitando as condições ambientais favoráveis ao desenvolvimento da planta, principalmente na época das chuvas. Como a cidade de Cruz das Almas tem um regime de chuvas mais regular, a população local não desenvolveu esse tipo de adaptação. Da mesma maneira, houve diferença significativa a $1 \%$ na análise de variância para IVG.

Apesar de pertencerem à mesma espécie, os indivíduos das duas populações apresentaram comportamentos germinativos diferentes. As diferenças adaptativas na germinação das duas populações de A. colubrina var. cebil evidenciaram que, com relação à germinação, essas duas populações estão bem adaptadas aos "habitats" em que ocorrem e ajudam a formar paisagens diversificadas, por meio de diferentes estratégias, contribuindo significativamente para o sucesso ecológico e evolutivo dessa espécie.

\section{AGRADECIMENTOS}

À Coordenadoria de Aperfeiçoamento do Pessoal de Ensino Superior (CAPES), pela bolsa de mestrado concedida a Rodrigues; ao Everaldo Mascarenhas Rodrigues, pelas correções no manuscrito; e ao Leonardo Neime Batista, pelas traduções.

\section{REFERÊNCIAS}

ALBUQUERQUE, U. P.; ANDRADE, L. H. C. Conhecimento botânico tradicional e conservação em uma área de caatinga no Estado de Pernambuco, Nordeste do Brasil. Acta Botanica Brasílica, v.16, n.3, p.273-285, 2002.
ANUÁRIO Estatístico da Bahia.

Disponível na internet via WWW.URL: http:// www.sei.ba.gov.br/publicacoes/bahia_dados/ anuario_estatistico/sumario/sum_2003.htm Arquivo capturado em 15 de março de 2005.

\section{BARBOSA, D. C. A. Estudos ecofisiológicos em Anadenanthera macrocarpa (Benth.) Brenan - aspectos de germinação e crescimento. 1980. $146 \mathrm{f}$. Tese (Doutorado em Ciências) - Universidade de São Paulo, São Paulo, 1980.}

BARBOSA, D. C. A. Distribution of Anadenanthera macrocarpa (Benth.) Brenan seedlings in an area of the caatinga of Northeastern Brazil. Boletim de Botânica, v.93, p.01-10, 1992.

BARBOSA, D. C. A. Estratégias de germinação e crescimento de espécies lenhosas da caatinga com germinação rápida. In: LEAL, I. R.; TABARELLI, M.; SILVA, J. M. C. (Ed.). Ecologia e conservação da caatinga. Recife: Universidade Federal de Pernambuco, 2003. p.625-656.

BORGHETTI, F.; FERREIRA, A. G. Interpretação de resultados de germinação. In: FERREIRA, A. G.; BORGHETTI, F. (Org.). Germinação - do básico ao aplicado. Porto Alegre: Artmed, 2004. p.209-222.

BOTEZELLI, L.; DAVIDE, A. C.; MALAVASI, M. M. Características dos frutos e sementes de quatro procedências de Dipteryx alata Vogel (baru). Cerne, v.6, n.1, p.09-18, 2000.

BRASIL. Ministério da Agricultura. Regras para análise de sementes. Brasília: SNDA/ LANARV, 1992. 188p.

CABRAL, E. L.; BARBOSA, D. C. A.; SIMABUKURO, E. A. Armazenamento e germinação de sementes de Tabebuia aurea (Manso) Benth. \& Hook. F. ex. S. Moore. Acta Botanica Brasílica, v.17, n.4, p.609-617, 2003.

CARVAlho, N. M.; NAKAGAWA, J. Sementes: ciência, tecnologia e produção. 4. ed. Jaboticabal: Funep, 2000. 588p. 
DHINGRA, O. D. et al. Seedborne pathogenic fungi that affect seedling quality of red angico (Anadenanthera macrocarpa) trees in Brazil. Journal of Phytopathology, v. 150, p.451455, 2002.

FERREIRA, A. G. et al. Germinação de sementes de Asteraceae nativas no Rio Grande do Sul, Brasil. Acta Botanica Brasílica, v.15, n.2, p.231-242, 2001.

HILHORST, H. W. M. et al. Curso avançado em fisiologia e tecnologia de sementes. Lavras: Universidade Federal de Lavras, 2001. 74p.

\section{LABORIAU, L. G. A germinação de} sementes. Washington: Secretaria Geral da Organização dos Estados Americanos, 1983. 174p.

MAGUIRE, J. D. Speed of germination - aid in selection aid evolution for seedling emergence and vigor. Crop Science, v.2, n.2, p.176-177, 1962.

NOBRE, S. A. M. Qualidade sanitária e fisiológica de sementes de ipê roxo (Tabebuia impetiginosa) e angico vermelho (Anadenanthera macrocarpa) em função de tratamentos diferenciados de frutos e sementes. 1994. 73f. Dissertação (Mestrado em Agronomia) - Escola Superior de Agricultura de Lavras, Lavras, 1994.

OLIVEIRA, D. M. T. Análise morfológica comparativa de frutos, sementes, plântulas e plantas jovens de 30 espécies arbóreas de Fabaceae ocorrentes no Estado de São Paulo. 1997. 212f. Tese (Doutorado em Biologia Vegetal) - Instituto de Biociências, Universidade Estadual Paulista, Rio Claro, 1997.

PIÑA-RODRIGUES, F. C. M.; FILGLIOLIA, M. B.; PEIXOTO, M. C. Testes de qualidade. In: FERREIRA, A. G.; BORGHETTI, F. (Org.). Germinação - do básico ao aplicado. Porto Alegre: Artmed, 2004. p.283-297.
RAMOS, A.; BIANCHETTI, A.; KUNIYOSKI, Y. S. Influência do tipo e da cobertura de canteiros na emergência e vigor de sementes de angico, Parapiptadenia rígida (Benth.) Brenan. Curitiba: Embrapa/URPFCS, 1982. p.81-87. (Documentos, 10).

RANIERI, B. D. et al. Germinação de sementes de Lavoisiera cordata Cogn. E Lavoisiera francavillana Cogn. (Melastomataceae), espécies simpátricas da Serra do Cipó, Brasil. Acta

Botanica Brasílica, v.17, n.4, p.523-530, 2003.

SANTANA, D. G.; RANAL, M. A. Análise estatística. In: FERREIRA, A. G.; BORGHETTI, F. (Org.). Germinação - do básico ao aplicado. Porto Alegre: Artmed, 2004. p.197-208.

SILVA, L. M. B. Ecofisiologia da reprodução e do crescimento de Anadenanthera macrocarpa (Benth.) Brenan (Mimosoideae) em uma área de caatinga, Alagoinha - PE. 1998. 86f. Dissertação (Mestrado em Biologia Vegetal) - Universidade Federal de Pernambuco, Recife: 1998.

SILVA, L. M. B.; BARBOSA, D. C. A. Crescimento e sobrevivência de Anadenanthera macrocarpa (Benth.) Brenan (Leguminosae), em uma área de caatinga, Alagoinha, PE. Acta Botanica Brasilica, v. 14, n.3, p.251-261, 2000.

SILVA, C. S. B. et al. Influência do substrato e da profundidade de semeadura na germinação de sementes de angico-de-caroço (Anadenanthera macrocarpa (Benth.) Brenan - Leguminosae) In: REUNIÃO NORDESTINA DE BOTÂNICA, 27. 2004, Petrolina. Anais... Petrolina: 2004.

VARELA,V.P.; COSTA, S.S.; RAMOS, M.B.P. Influência da temperatura e do substrato na germinação de sementes de itaubarana (Acosmium nitens (Vog.) Yakovlev) Leguminosae, Caesalpinoideae. Acta Amazonica, v.35, n.1, p.35-39, 2005. 